\title{
Article \\ Multi-Scale Nonlinear Progressive Damage and Failure Analysis for Open-Hole Composite Laminates
}

\author{
Jian Shi * and Mingbo Tong *
}

check for updates

Citation: Shi, J.; Tong, M. Multi-Scale Nonlinear Progressive Damage and Failure Analysis for Open-Hole Composite Laminates. Aerospace 2022, 9,59. https://doi.org/10.3390/ aerospace 9020059

Academic Editor: Stelios K. Georgantzinos

Received: 29 December 2021 Accepted: 19 January 2022

Published: 24 January 2022

Publisher's Note: MDPI stays neutral with regard to jurisdictional claims in published maps and institutional affiliations.

Copyright: (C) 2022 by the authors. Licensee MDPI, Basel, Switzerland. This article is an open access article distributed under the terms and conditions of the Creative Commons Attribution (CC BY) license (https:// creativecommons.org/licenses/by/ $4.0 /)$.
State Key Laboratory of Mechanics and Control of Mechanical Structures, Nanjing University of Aeronautics and Astronautics, Nanjing 210016, China

* Correspondence: shijian@nuaa.edu.cn (J.S.); tongw@nuaa.edu.cn (M.T.)

\begin{abstract}
In order to study the nonlinear behaviors and interactions among the constituents for the composite material structure under the tensile load, multiscale damage model using generalized method of cells (GMC) and a lamina-level progressive damage model were established, respectively, for fiber reinforced composite laminates with a central hole, which were based on the thermodynamic Schapery Theory (ST) at either the micro-level or the lamina level. Once the nonlinear progressive degradation of the matrix material reached the lower limit value for the ST method, matrix failures naturally occurred, the failure of the fiber was determined by the maximum stress failure criterion. For the multiscale progressive damage model, the GMC model consisting of a fiber subcell and three matrix subcells was imposed at each integral point of FEM elements, and the three matrix subcells undergo independent damage evolution. The load versus displacement curves and failure modes of the open-hole laminates were predicted by using the two progressive failure models, and the results were compared with that obtained by the Hashin-Rotem progressive failure model and the experimental results. The results show that the ST based method can obtain the nonlinear progressive damage evolution states and failure states of the composite at both the lamina level and the multiscale level. Finally, the damage contours and failure paths obtained are also presented.
\end{abstract}

Keywords: progressive damage analysis; generalized method of cells; multi-scale analysis; schapery theory; fiber reinforced composite

\section{Introduction}

Polymer matrix composites (PMCs) have been widely used in aircraft structures owing to their low weight, high strength, high resistance to fatigue, and many other superior advantages. Progressive damage analysis of composite laminates is regarded as an important and complex subject, which is highlighted by the World-Wide Failure Exercise (WWFE) [1-3]. It is mainly based on macroscopic failure analysis methods within the WWFE to predict the final failure behavior of fiber reinforced composites. The associated parameters in these theories generally rely on extensive mechanical experiments, which result in myriad costs of time and expense. Although many criteria take different failure modes into account and incorporate progressive failure modeling [4-6], they are essentially phenomenological methods that cannot capture the interaction between the constituents at the microscale level. In order to more accurately characterize the mechanical response of the fiber reinforced composite structures, damage and failure mechanisms must be treated separately [7-9]. The gradual expansion of matrix micro-damage or micro-crack originates from the distributed micro-pores or other micro-defects produced in the manufacturing of composite structures. The damage expansion leads to stiffness reduction in local areas of the structure, resulting in redistribution of stress and strain fields, which is the main cause of nonlinear phenomena before the catastrophic failure of fiber reinforced composite materials occurs [10]. 
Progressive damage and failure analysis of composite structures with an open hole is one of the hot subjects for structural designs [11,12]. Stress concentration and redistribution around the open hole in a laminate subjected to tensile loads can lead to produce complicated damage and failure modes [13], including matrix cracking, fiber breakage, delamination, etc. $[14,15]$. Linear elasticity along with different failure criteria are usually employed to predict the damage and failure states of composite structures with an open hole for many analytical and numerical models at the micro level [16]. Even though the failure of fiber and matrix is treated distinctly for these progressive damage methods [6], it is not available to obtain the interaction between the constituents.

Micromechanical models are able to acquire the quantitative relationship among the constituent materials within the composites [17]. By using microscopic mechanical methods, variations of constituent material properties, constituent phases or the microscopic geometries, such as the fiber volume fraction and micro-level architecture, can be considered conveniently to promote a further study of the failure mechanism for composite structures. The generalized method of cells (GMC) $[18,19]$ based on a homogenization technique are actually analytical, which has been proved to have very high computational efficiency. Formulations of the GMC model contain the applications of some governing equations using a homogenization method, by which traction and displacement continuity equations are imposed at the subcell interfaces and the periodic boundaries of the repeating unit cell (RUC) in an integral sense. It is convenient to apply the micromechanics model GMC to the multiscale analysis within commercial finite element software [20,21].

The main objective of the current work is to use a thermodynamically based Schapery Theory (ST) [22-24] to predict the nonlinear response, the damage evolution, and the failure contour for the composite laminates with an open hole under tensile loading. Two progressive damage methods are used, one is ST/FF (Schapery Theory/Fiber Failure) method at the laminate level, the other is a multi-scale analysis method ST/GMC/FF (Schapery Theory/Generalized Method of Cells/Fiber Failure) at micro-scale level, both models contain a ST based degradation strategy of elastic properties for the matrix damage and the maximum stress failure criterion for the fiber failure, which are employed at either the lamina level or the micro level. The results obtained from these two models are compared with the experimental data and that analysed using Hashin-Rotem progressive damage method embedded in the ABAQUS software.

\section{Multiscale Method of Progressive Damage and Failure}

\subsection{ST Model}

The Schapery Theory can be used for the progressive damage analysis of the fiber reinforced composites, in which the total applied potential $W_{T}$ of the material is divided into the recoverable elastic part $W$ and the dissipative part $W_{S}$ that can cause structural deformation [25].

$$
W_{T}=W+W_{S}
$$

A portion of the total applied work potential causes the microcracking and any other structural changes when the material is loaded, which affects the elastic properties of the material, and at the same time, the other portion of the applied work potential can be recovered when the material is unloaded. This process is shown in Figure 1, where the shaded area represents the dissipated potential $W_{S}$ and the area below the straight line represents the elastic potential $W$.

According to the ST method [22,23], both $W$ and $W s$ are functions of a group of internal state variables (ISVs) $S_{m}(m=1,2, \ldots, \mathrm{M})$. The total applied work potential is a constant with respect to each of the internal state variables [23],

$$
\frac{\partial W_{T}}{\partial S_{m}}=0
$$




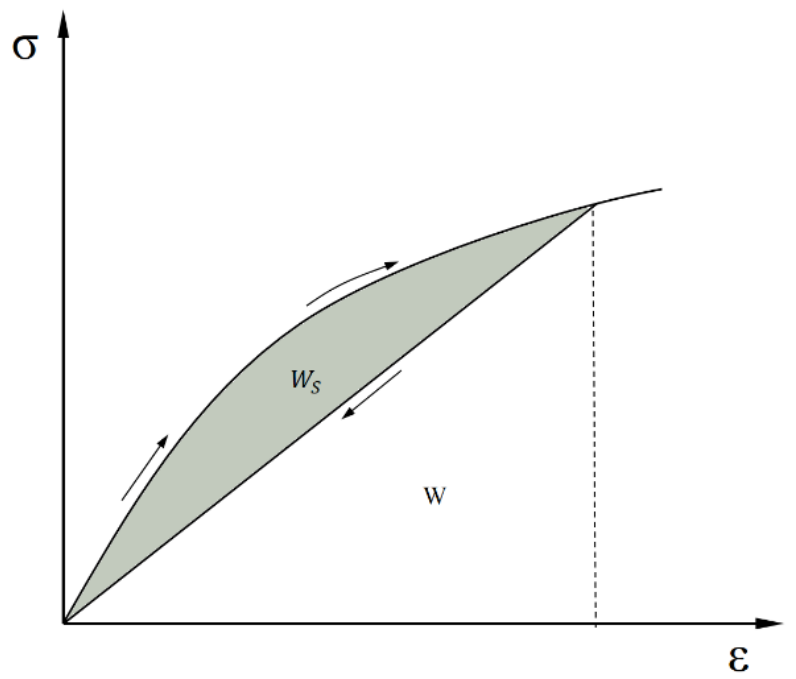

Figure 1. Irrecoverable portion $W s$ (shaded area) and the elastic portions $W$.

The dissipated potential $W_{S}$ is allowed to be a function of any number of internal state variables, and different internal state variables can be used to explain different damage and failure mechanisms. In order to describe the nonlinear progressive damage of the matrix material of the fiber reinforced composites, Ws is assumed to be a function of a single internal variable $S$ [25]. For convenience, $W s=S$ can be selected,

$$
W_{T}=W+S
$$

Calculating the derivative of Equation (3) with respect to $S$ yields,

$$
\frac{\partial W}{\partial S}=-1
$$

\subsection{Constitutive Relation}

The constitutive relation of a unidirectional lamina under the plane stress state can be written as,

$$
\begin{gathered}
\sigma_{11}=Q_{11} \varepsilon_{11}+Q_{12} \varepsilon_{22} \\
\sigma_{22}=Q_{12} \varepsilon_{11}+Q_{22} \varepsilon_{22} \\
\tau_{12}=Q_{66} \gamma_{12}
\end{gathered}
$$

where $\gamma_{12}$ is the engineering shear strain, and

$$
\begin{gathered}
Q_{11}=\frac{E_{11}}{1-v_{11} v_{21}} \\
Q_{22}=\frac{E_{22}}{1-v_{12} v_{21}} \\
Q_{12}=v_{12} Q_{22} \\
Q_{66}=G_{12} \\
v_{21}=\frac{v_{12} E_{22}}{E_{11}}
\end{gathered}
$$

where $E_{11}, E_{22}, v_{12}, v_{21}$, and $G_{12}$ denote axial elastic modulus, transverse elastic modulus, Poisson's ratio, transverse Poisson's ratio, and shear modulus respectively. It can be assumed that $v_{12} v_{21} \ll 1$, then Equation (6) becomes

$$
\begin{gathered}
Q_{11}=E_{11} \\
Q_{22}=E_{22} \\
Q_{12}=v_{12} Q_{22} \\
Q_{66}=G_{12}
\end{gathered}
$$




\subsection{Calculating the Damage State}

The damage mechanism associated with the internal state variable $S$ only is limited to the matrix phase of composite materials, so it is considered that the damage is limited to $E_{22}$ and $G_{12}$, or only degrades the matrix material in the RUC. Both $E_{22}$ and $G_{12}$ are functions of the internal state variable $S$, which can be expressed as,

$$
\begin{aligned}
& E_{22}=E_{220} e_{s}(S) \\
& G_{12}=G_{120} g_{s}(S)
\end{aligned}
$$

where, $E_{220}$ and $G_{120}$ represent the original transverse elastic modulus and shear modulus, and $e_{S}(S)$ and $g_{S}(S)$ represent the polynomial function of the transverse and shear modulus with respect to $S$.

According to the plane stress constitutive relation, the expression of elastic strain energy density $W$ can be expressed as,

$$
W=\frac{1}{2}\left(E_{11} \varepsilon_{11}^{2}+E_{22} \varepsilon_{22}^{2}+G_{12} \gamma_{12}^{2}\right)+Q_{12} \varepsilon_{11} \varepsilon_{22}
$$

By assuming $Q_{12}$ is constant, and differentiating Equation (10) with respect to $S$ yields,

$$
\frac{\varepsilon_{22}^{2}}{2} \frac{\partial E_{22}}{\partial S}+\frac{\gamma_{12}^{2}}{2} \frac{\partial G_{12}}{\partial S}=-1
$$

Experiments show that variable $S$ is a function of $\varepsilon^{3}$ [25], so a reduced internal state variable $S_{\gamma}$ can be introduced,

$$
S=S_{\gamma}^{3}
$$

Then, Equation (11) becomes

$$
\frac{\varepsilon_{22}^{2}}{2} \frac{\partial E_{22}}{\partial S_{\gamma}}+\frac{\gamma_{12}^{2}}{2} \frac{\partial G_{12}}{\partial S_{\gamma}}=-3 S_{\gamma}^{2}
$$

Once $S_{\gamma}$ is solved by Equation (13), the elastic moduli $E_{22}$ and $G_{12}$ can undergo nonlinear degradation according to Equations (8) and (9).

\subsection{Generalized Method of Cells}

The generalized method of cells (GMC) is employed to predict the strengths of continuous fiber composites in this work. The fiber reinforced composite is modeled as a rectangular repeating unit cell that contains a preset number of rectangular subcells as shown in Figure 2, and each subcell $(\beta \gamma)$ may be occupied by a distinct homogeneous material. The constitutive equation in each subcell for the micromechanical model is denoted below,

$$
\sigma^{(\beta \gamma)}=C^{(\beta \gamma)} \mathcal{\varepsilon}^{(\beta \gamma)}
$$

where $\sigma^{(\beta \gamma)}, C^{(\beta \gamma)}$, and $\varepsilon^{(\beta \gamma)}$ represent the average stress components, the elastic stiffness matrix, and the average total strain components in subcell $(\beta \gamma)$, respectively. Inelastic strain and thermal strain are not took into considered herein.

The basic displacement assumptions in GMC is a linear polynomial, as shown in Formula (15), with the local coordinates $\left(\bar{x}_{2}^{(\beta)}, \bar{x}_{3}^{(\gamma)}\right)$ located at the center of each subcell,

$$
u_{i}^{(\beta \gamma)}=W_{i(00)}^{(\beta \gamma)}+\bar{y}_{2}^{(\beta)} W_{i(10)}^{(\beta \gamma)}+\bar{y}_{3}^{(\gamma)} W_{i(01)}^{(\beta \gamma)} i=1,2,3
$$

where $W_{i(00)}^{(\beta \gamma)}$ represents the displacements at the center of subcell $(\beta \gamma)$, microvariables $W_{i(10)}^{(\beta \gamma)}$ and $W_{i(01)}^{(\beta \gamma)}$ characterize the first-order dependence of the displacement field on the local coordinates $\bar{x}_{2}^{(\beta)}$ and $\overline{\boldsymbol{x}}_{3}^{(\gamma)}$. 


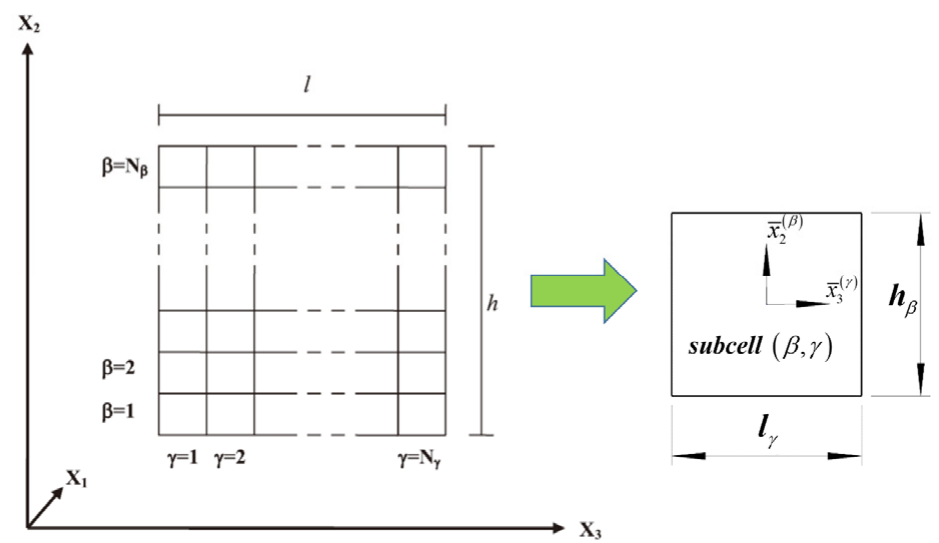

Figure 2. Discretization of the repeating unit cell.

The formulation of GMC involves imposition of several governing conditions, including application of continuity of displacements and tractions at each of the subcell interfaces and the periodic boundaries of the repeating unit cell (RUC) in an average sense. By regarding the stress components in the subcells as the basic unknowns, a system of equations relating the unknown stress variables to the macroscopic strains can be represented as follows,

$$
\mathbf{G T}=\mathbf{f}^{m}
$$

where the $\mathbf{G}$ matrix consists of information on the geometry and mechanical properties of the material in the individual subcells $(\beta \gamma)$, the $\mathbf{T}$ vector represents the subcell stress components that need to be solved, and the $\mathbf{f}^{m}$ vector incorporates information of geometrical dimensions and the global strains on the RUC.

The expression that relates the average strain in the subcell to the externally global strain in virtue of the solutions of Equation (16), then the final form of the global constitutive equation that relates the average stress $\bar{\sigma}$ and strain $\bar{\varepsilon}$ is determined as follow,

$$
\bar{\sigma}=C^{*} \bar{\varepsilon}
$$

where $C^{*}$ is the average or effective stiffness matrix.

The global stress can be established in the GMC repeating unit cell as follow,

$$
\bar{\sigma}=\frac{1}{h l} \sum_{\beta=1}^{N_{\beta}} \sum_{\gamma=1}^{N_{\gamma}} h_{\beta} l_{\gamma} \sigma^{(\beta \gamma)}
$$

where $h$ and $l$ represent the height and length of the RUC, $h_{\beta}$ and $l_{\gamma}$ represent the height and length of the subcell $(\beta \gamma)$, as shown in Figure 2.

The GMC model is applied to each integration point of each finite element, and the strain states of each integral point are taken as the load input to the RUC, then the stress and strain states of each constitutive materials can be obtained according to Equation (16). The damage evolution and failure are evaluated at the micro-level, and after updating the stiffness matrix using the homogenization method, the multi-scale analysis will entry the next finite element iterative calculation. Figure 3 is a schematic diagram of the analysis process. 


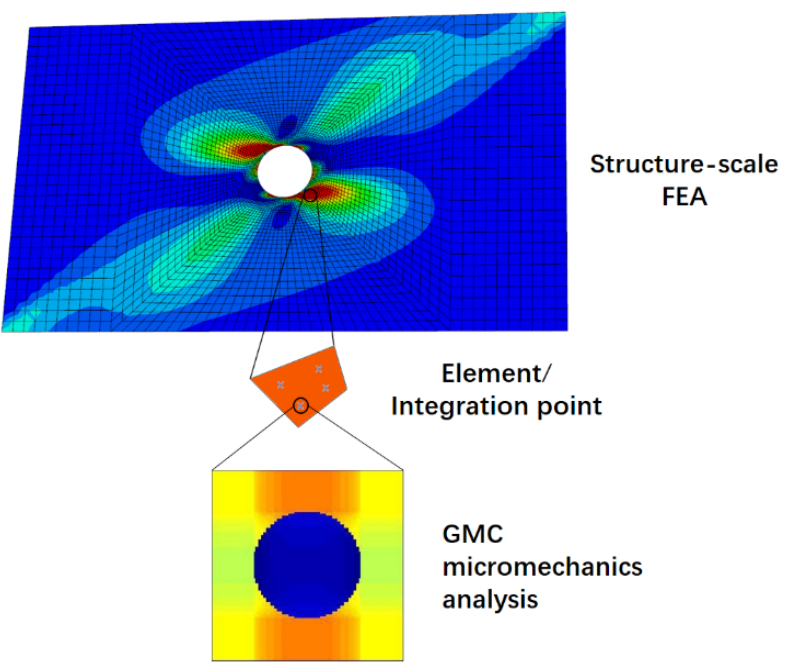

Figure 3. FEM with generalized method of cells (GMC).

\section{Analysis Method}

Three analysis methods are adopted in this paper, one is a multi-scale analysis method based on GMC, the second method is based on ST at the lamina level, and the last one employs an empirical method based on the 2D Hashin-Rotem failure criteria and implicit solver of static analysis is used. The maximum stress failure criterion for fiber subcells and ST theory for matrix subcells are employed for the first method, the maximum stress failure criterion together with a degradation limit are used for the second method. Once the reduced internal state variable $S_{\gamma}$ is determined, the transverse and shear stiffness of matrix properties degrades according to the damage functions $e_{s}$ and $g_{s}$. These damage and failure models for the static analysis are all implemented in ABAQUS/Standard employing the user-defined subroutine UMAT.

\subsection{Lamina Level Damage and Failure Model Based on ST}

According to the experimental data of transverse elastic modulus $E_{22}$ and shear modulus $G_{12}$ corresponding to internal variable $S_{\gamma}$ in literature [26], damage functions $e_{S}$ and $g_{\mathrm{s}}$ are obtained by cubic polynomial fitting, as shown in equations below. The coefficients are shown in Table 1 . Figure 4 shows the polynomial $e_{\mathrm{s}}$ and $g_{\mathrm{s}}$ obtained by fitting and the experimental data, respectively. The fitting effect is very ideal. The damage evolution rate of $G_{12}$ is obviously higher than that of $E_{22}$.

$$
\begin{gathered}
e_{s}=e_{s 0}+e_{s 1} S_{\gamma}+e_{s 2} S_{\gamma}^{2}+e_{s 3} S_{\gamma}^{3} \\
g_{s}=g_{s 0}+g_{s 1} S_{\gamma}+g_{s 2} S_{\gamma}^{2}+g_{s 3} S_{\gamma}^{3}
\end{gathered}
$$

Table 1. Micro-damage polynomial coefficients for $E_{22}$ and $G_{12}$.

\begin{tabular}{cccc}
\hline $\begin{array}{c}\boldsymbol{E}_{\mathbf{2 2}} \\
\text { Coefficients }\end{array}$ & Values & $\begin{array}{c}\boldsymbol{G}_{\mathbf{1 2}} \\
\text { Coefficients }\end{array}$ & Values \\
\hline$e_{S 0}$ & 1 & $g_{S 0}$ & 1 \\
$e_{S 1}$ & -0.1308 & $g_{S 1}$ & -0.7269 \\
$e_{S 2}$ & -0.5650 & $g_{S 2}$ & -0.6706 \\
$e_{S 3}$ & 0.2304 & $g_{S 3}$ & 0.3932 \\
\hline
\end{tabular}




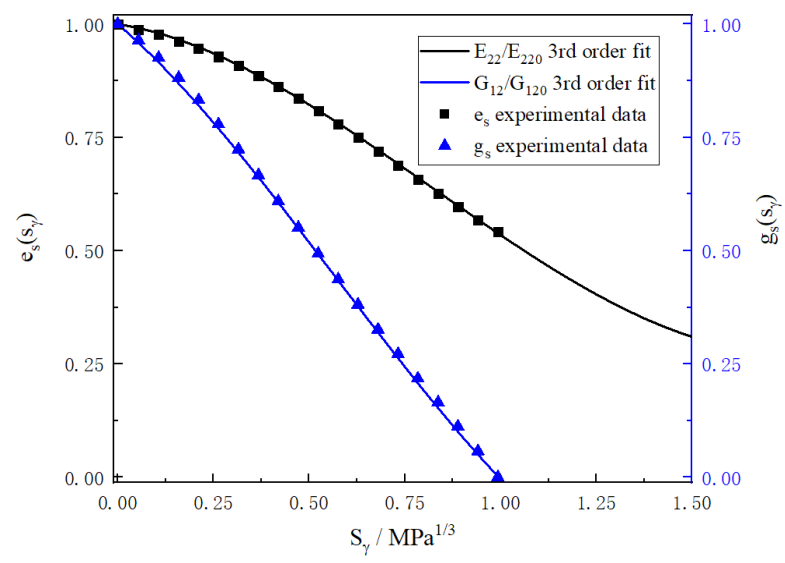

Figure 4. Ratio of actual modulus to virgin modulus as a function of the reduced internal state variable.

According to Equation (13), the value of $S_{\gamma}$ depends on the strain state of the repeating unit cell, that is, it is determined by the transverse strain $\varepsilon_{22}$ and shear strain $\gamma_{12}$. Figure 5 show the changing curves of $S_{\gamma}$ with $\varepsilon_{22}$ in the interval [0, 0.035] when $\gamma_{12}$ is selected to be $0,0.0025,0.005,0.0075$, and 0.01 . It can be seen that the positive solution of $S_{\gamma}$ is unique and monotonically increasing. The growth rate increases first, then decreases, and finally flattens out. The values of $S_{\gamma}$ are between 0 and 1.2.

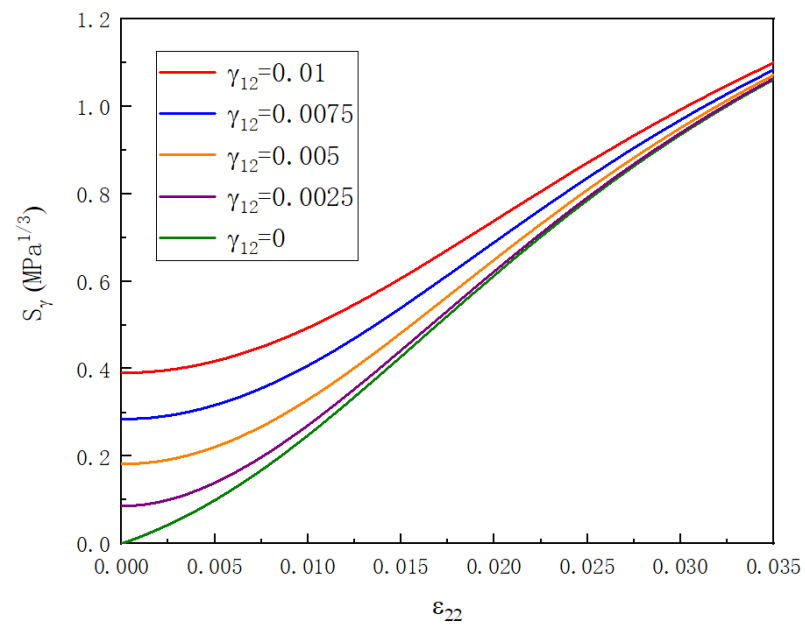

Figure 5. Changes of the reduced internal state variable with strain states.

It is generally believed that fiber fracture occurs instantaneously and results in immediate local failure of laminates. Therefore, the properties of fiber is deemed to be linear before it reaches the ultimate failure, so the strength prediction can be satisfied by using a simple maximum stress failure criterion.

$$
\begin{aligned}
& \left(\frac{\sigma_{11}}{X_{T}}\right)^{2}=d_{f}^{2}, \sigma_{11}>0 \\
& \left(\frac{\sigma_{11}}{X_{C}}\right)^{2}=d_{f}^{2}, \sigma_{11}<0
\end{aligned}
$$

where $\sigma_{11}$ is the stress in the fiber direction, $X_{T}$ and $X_{C}$ are the fiber direction tensile and compressive strengths. Fiber failure will initiate when $d_{f}$ exceed 1.

For the matrix damage of monolayer plate, the degradation of $E_{22}$ and $G_{12}$ for a laminate or the RUC is reduced by Equations (8) and (9). Since material softening may cause convergence problems for the finite element model using the implicit algorithm, the degradation ratio of $E_{22}$ and $G_{12}$ was limited to 0.4 , after which the damage evolution was 
terminated and $E_{22}$ and $G_{12}$ would remain constant, and the matrix material is considered to be failed.

\subsection{Micro-Scale Damage and Failure Model}

The micromechanical GMC model can capture the interaction between the constituents of composite materials, and can easily analyze the damage or failure of fiber and matrix phases independently. Each material point in the finite element model corresponds to a $2 \times 2$ RUC [21], as shown in Figure 6, containing one fiber subcell and three matrix subcells. The fiber volume fraction of T800/3900-2 lamina is 0.6, and its properties of fiber and matrix constituents are shown in Tables 2 and 3.

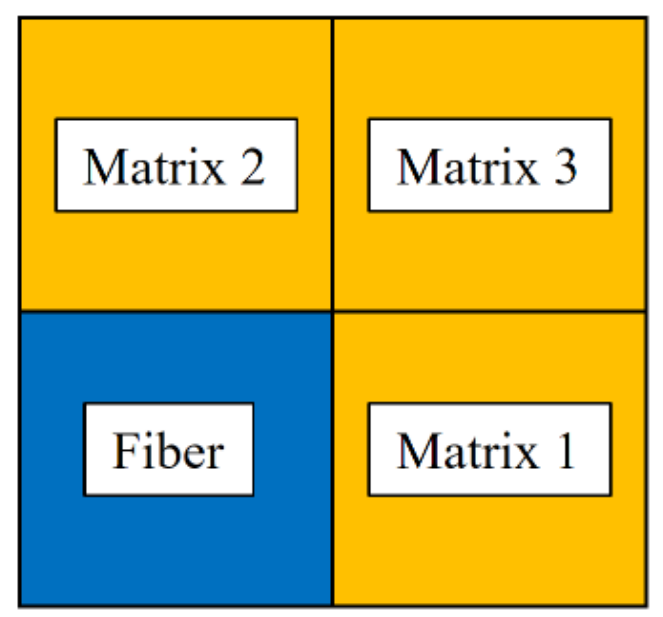

Figure 6. $2 \times 2$ RUC used in micromechanics simulations.

Table 2. Elastic properties of the composite constituents.

\begin{tabular}{cccc}
\hline $\begin{array}{c}\text { Fiber } \\
\text { Properties }\end{array}$ & Values/GPa & $\begin{array}{c}\text { Matrix } \\
\text { Properties }\end{array}$ & Values/GPa \\
\hline$E_{f 11}$ & 293 & $E_{m 11}$ & 2350 \\
$E_{f 22}$ & 91 & $E_{m 22}$ & 2350 \\
$v_{f 12}$ & 0.23 & $v_{m 12}$ & 0.35 \\
$v_{f 23}$ & 0.45 & $v_{m 23}$ & 0.35 \\
$G_{f 12}$ & 55 & $G_{m 12}$ & 2300 \\
\hline
\end{tabular}

Table 3. Strengths of the composite constituents.

\begin{tabular}{cc}
\hline Properties & Values/MPa \\
\hline Tensile fiber strength, $X_{f t}$ & 5200 \\
Compressive fiber strength, $X_{f c}$ & 2855 \\
Matrix transverse tensile strength, $Y_{m t}$ & 49 \\
Matrix transverse compressive strength, $Y_{m c}$ & 124 \\
Matrix shear strength, $S$ & 41 \\
\hline
\end{tabular}

Fiber subcells are considered to be linear before the failure occurs, and the fiber subcell failure is determined by the maximum stress failure criterion, which is shown in Equations (23) and (24). The material properties of fiber constituent after failure are immediately reduced to $0.01 \%$ of its initial value.

$$
\left(\frac{\bar{\sigma}_{11}^{(11)}}{X_{f t}}\right)^{2}=d_{f}^{2}, \bar{\sigma}_{11}>0
$$




$$
\left(\frac{\bar{\sigma}_{11}^{(11)}}{X_{f c}}\right)^{2}=d_{f}^{2}, \bar{\sigma}_{11}<0
$$

where $\bar{\sigma}_{11}^{(11)}$ is the stress of the fiber subcell in the longitudinal direction, $X_{f_{c}}$ and $X_{f c}$ are its tensile and compressive strengths. When damage variable $d_{f}$ is greater than or equal to 1 , fiber subcells would fail.

In each time step of the multiscale analysis, the GMC micromechanics model calculates the overall elastic moduli should be consistent with the laminate level moduli obtained from Equations (8) and (9). Therefore, the matrix subcells should be degraded in a certain way in order to produce the same $E_{22}$ and $G_{12}$. Matrix constituent properties are also functions of the reduced internal state variable $S_{\gamma}$,

$$
\begin{aligned}
& E_{m}=E_{m 0} e_{s}\left(S_{\gamma}\right) \\
& G_{m}=G_{m 0} g_{s}\left(S_{\gamma}\right)
\end{aligned}
$$

The value of $S_{\gamma}$ is determined by the following formula,

$$
\frac{\left(\bar{\varepsilon}_{22}^{(\beta \gamma)}\right)^{2}}{2} \frac{\partial E_{m 0}}{\partial S_{\gamma}}+\frac{\left(\bar{\gamma}_{12}^{(\beta \gamma)}\right)^{2}}{2} \frac{\partial G_{m 0}}{\partial S_{\gamma}}=-3 S_{\gamma}^{2}
$$

where $\bar{\varepsilon}_{22}^{(\beta \gamma)}$ and $\bar{\gamma}_{12}^{(\beta \gamma)}$ represent transverse strain and shear strain of matrix subcell $(\beta \gamma)$. Matrix subcell stress vector $\bar{\sigma}^{(\beta \gamma)}$ can be obtained by the following formula,

$$
\bar{\sigma}^{(\beta \gamma)}=A \bar{\varepsilon},
$$

$A$ is a third-order matrix connecting the macroscopic strain components and the stress components of each subcell under the plane stress state.

Using the constitutive relation of the subcell material, there is

$$
\bar{\varepsilon}^{(\beta \gamma)}=S^{(\beta \gamma)} \bar{\sigma}^{(\beta \gamma)}
$$

where $S^{(\beta \gamma)}$ is the flexibility matrix of the subcell $(\beta \gamma)$ material.

Combined with Equations (28) and (29), the expressions of strain of each subcell are as follows:

$$
\bar{\varepsilon}^{(\beta \gamma)}=S^{(\beta \gamma)} A \bar{\varepsilon}
$$

Due to the different stress-strain states of each matrix subcell at the same material point in the analysis process, the damage evolution rates of different matrix subcells are different. In order to keep the same as the damage limit 0.4 of lamina level $E_{22}$ and $G_{12}$, the matrix subcell material with damage evolution dropping to 0.4 earlier stops, degrading until the last matrix subcell reaches the degradation limit, which represents the final matrix failure of the RUC. The flow chart of multi-scale analysis algorithm based on a four-subcells GMC model is as shown in Figure 7.

\subsection{Lamina-Scale Failure Model-Based Hashin-Rotem Theory}

The Hashin-Rotem failure theory include separate criteria for fiber and matrix. The fiber failure criteria are the same as the maximum stress criteria as shown in Equations (21) and (22). Matrix failure are contributed by the transverse and shear stresses in a lamina, which are dictated by

$$
\begin{aligned}
& \left(\frac{\sigma_{22}}{Y_{T}}\right)^{2}+\left(\frac{\tau_{12}}{S}\right)^{2}=d_{\mathrm{m}}^{2}, \sigma_{22}>0 \\
& \left(\frac{\sigma_{22}}{Y_{C}}\right)^{2}+\left(\frac{\tau_{12}}{S}\right)^{2}=d_{\mathrm{m}}^{2}, \sigma_{22}<0
\end{aligned}
$$


where $\sigma_{22}$ and $\tau_{12}$ are the transverse and shear stresses in a lamina, $Y_{T}, Y_{C}$, and $S$ are respectively the transverse compressive strength, transverse tensile strength, and shear strength in the composite lamina.

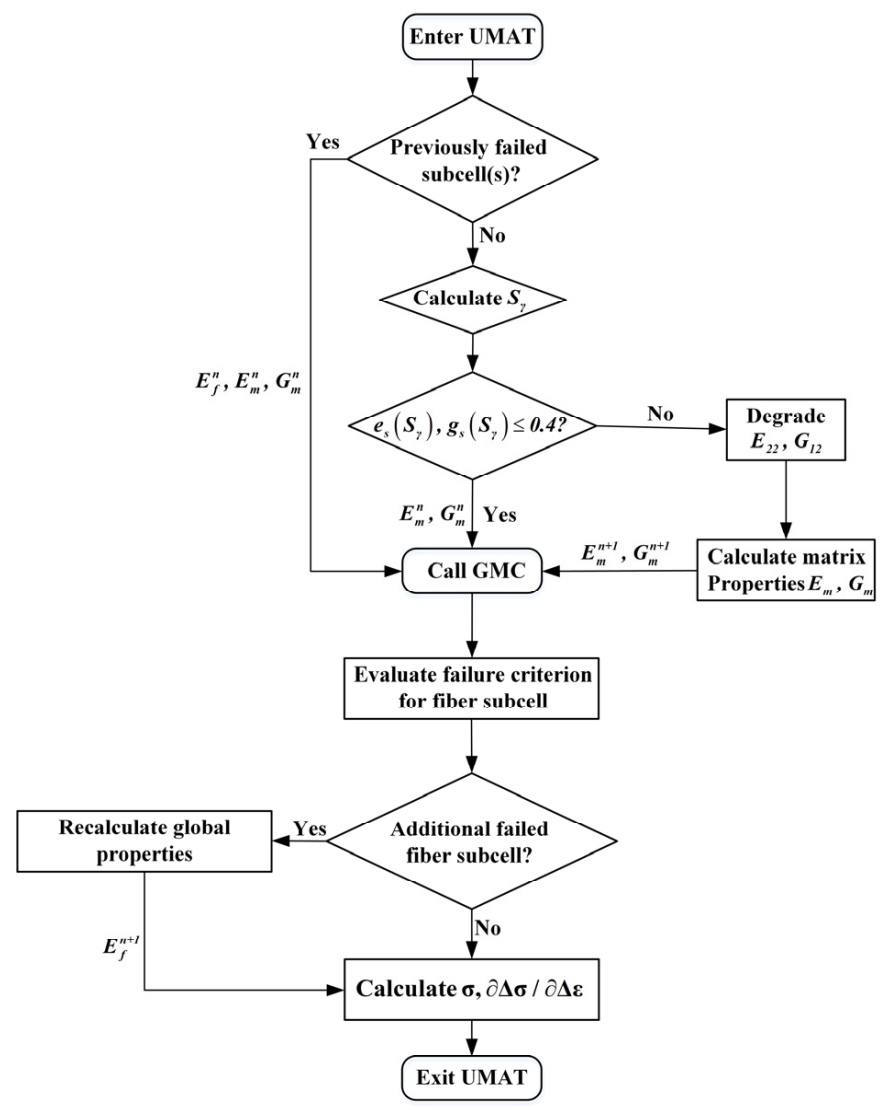

Figure 7. Flow chart of hierarchy for multiscale algorithm with damage and failure at the micro-scale.

\section{Results and Discussion}

\subsection{Experimental Result}

A total of 8 composite specimens with a central opening hole are provided, of which the geometric dimensions are: $250 \mathrm{~mm}$ long, $25.26 \mathrm{~mm}$ wide, $2.28 \mathrm{~mm}$ thick, and the central hole diameter $4.76 \mathrm{~mm}$. [45/ $-45 / 0 /-45 / 0 / 45 / 45 / 0 /-45 / 0 /-45 / 45]$ are the laminate stacking sequences, and the material properties of these specimens correspond to T800/3900-2 as shown in Table 4.

Table 4. Elastic properties of the T800/3900-2 lamina.

\begin{tabular}{cccc}
\hline $\begin{array}{c}\text { Elastic } \\
\text { Properties }\end{array}$ & Values & $\begin{array}{c}\text { Elastic } \\
\text { Properties }\end{array}$ & Values \\
\hline$E_{1} / \mathrm{GPa}$ & 154 & $\mathrm{G}_{23} / \mathrm{MPa}$ & 4340 \\
$E_{2} / \mathrm{GPa}$ & 9 & $E_{m 12}$ & 0.29 \\
$E_{3} / \mathrm{GPa}$ & 9 & $v_{m 13}$ & 0.29 \\
$G_{12} / \mathrm{MPa}$ & 4340 & $v_{m 23}$ & 0.45 \\
$G_{13} / \mathrm{MPa}$ & 4340 & & \\
\hline
\end{tabular}

The tensile test process of open-hole laminates is conducted in accordance with ASTM D5766 — standard test method for open-hole tensile strength of polymer matrix composite laminates. The open-hole tensile test of laminates was carried out on MTS 370 electrohydraulic servo test machine, which has a maximum load-carrying capacity of $250 \mathrm{kN}$ with a accuracy of $0.5 \%$. The displacement load curves of the test pieces are shown in Figure 8 . 


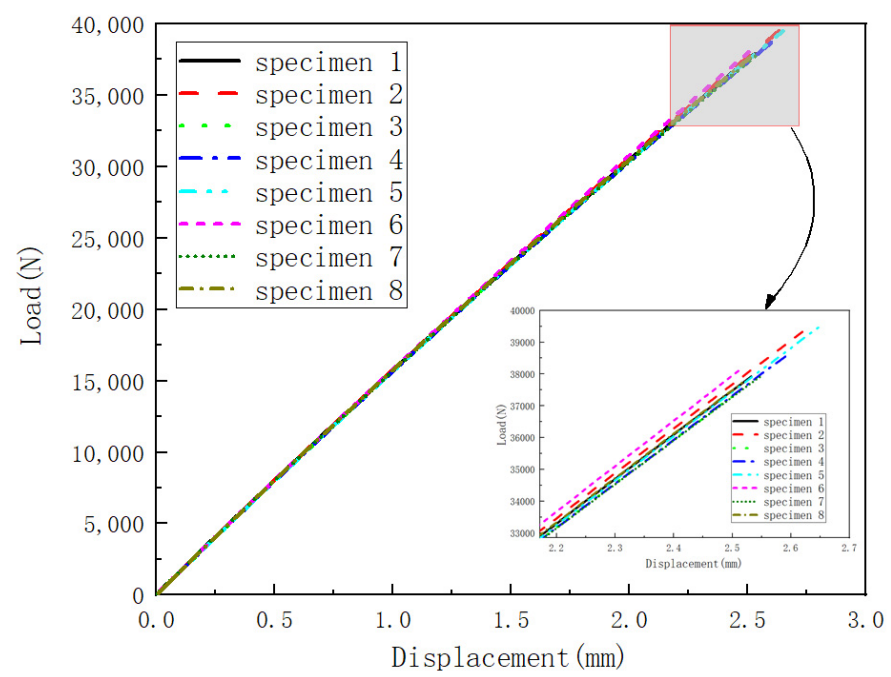

Figure 8. The force-displacement curve of eight specimens.

The average failure load of all specimens is $38.34 \mathrm{kN}$, and the dispersion coefficient is $2.13 \%$. It can be seen from the displacement versus load curve that all the specimens are obviously brittle before the ultimate failure determined by the tensile strength of $0^{\circ}$ layer onset. The photograph of the failed specimen is present in Figure 9, it can be observed that the catastrophic failures are concentrated around the open hole which causes the stress concentrations and finally span the width of the specimen, the failure modes of the specimen are mainly as follows: tensile fracture of fiber for $0^{\circ}$ layer and tensile fracture of the matrix for $\pm 45^{\circ}$ layer.

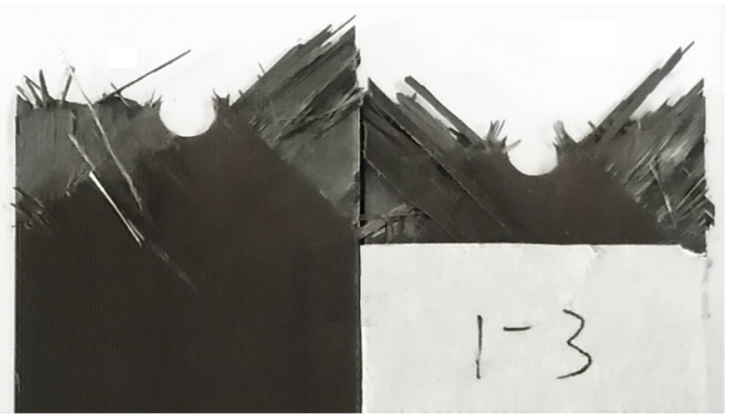

Figure 9. Photograph of failed laminate specimen.

\subsection{Results of Finite Element Analysis}

The three methods implemented using the subroutine UMAT introduced in Section 2 above are used to conduct the finite element analysis of the open-hole laminate specimens. The S4R mesh type is used, and the mesh is refined around the open hole. The composite constituents properties can be seen in Tables 2 and 3, and the laminate properties are shown in Table 4. Additionally, the tensile and compressive strengths of the composite laminate are $2690 \mathrm{MPa}$ and $1380 \mathrm{MPa}$, respectively, which are provided by vendors and used to perform FEA at the micro-level. The finite element model is restricted from moving in the thickness direction and all the in-plane rotational degrees of freedom to account for plane stress simulation. The left end of the model is fixed and the right edge is constrained in the transverse displacement and all rotations, then a tensile displacement of $5 \mathrm{~mm}$ is applied to the right end. The load versus displacement curves obtained from the three progressive damage methods are shown in Figure 10. It can be observed that the ultimate load prediction for ST / FF (38.76 kN), Hashin-Rotem $(39.49 \mathrm{kN})$ and ST/GMC / FF (38.93 kN) agree well with the experimental value $(38.45 \mathrm{kN})$. The load displacement curve obtained by Hashin-Rotem progressive damage method is linear, but both ST/FF and ST/GMC 
methods achieve satisfactory nonlinear reduction before the displacement-load curve reaches its peak. The load displacement curve obtained in the experiment has shown, to some extent, nonlinear behavior, especially near the end of the experimental curve. In the experiment, the crack initiates around the open hole and propagates rapidly to the edge of the specimen. It is not possible to capture the rapid progression of the crack by using the implicit solver, so the gradual failure of fiber and matrix yield the exhibited softening in the ST or ST/GMC model.

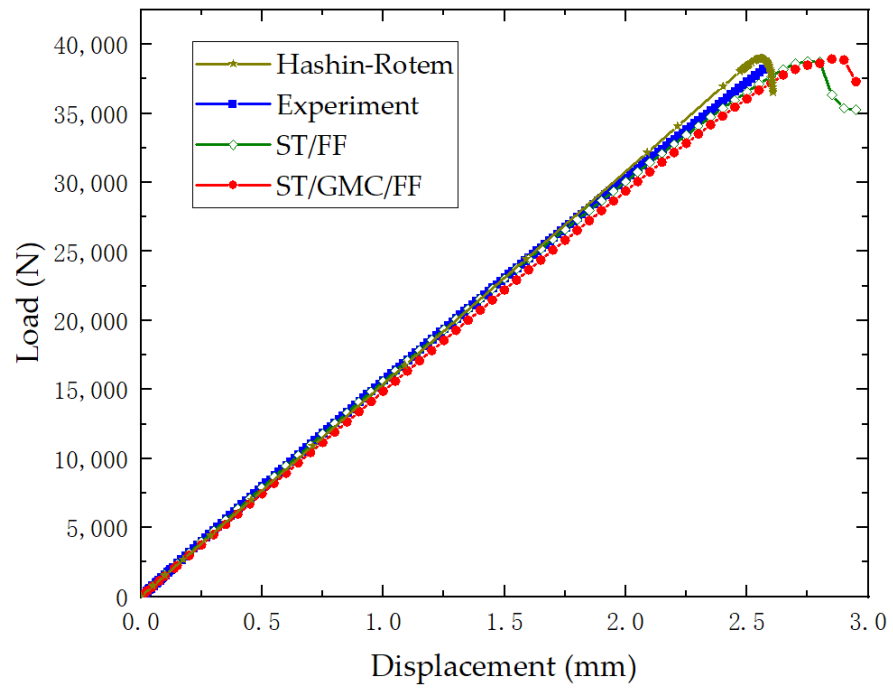

Figure 10. The force-displacement curve of simulations.

Figure 11 shows the fiber damage and matrix nonlinear damage state of $0^{\circ}$ layer and $45^{\circ}$ layer under different loads by ST/FF method. As the damage states of the $-45^{\circ}$ layer and $45^{\circ}$ layer are basically symmetric with respect to the $0^{\circ}$ axis, the damage contour of $0^{\circ}$ layer and $45^{\circ}$ layer are selected to be shown below. At $80 \%$ ultimate load, tensile and shear damage begin to fail at the upper and lower edges of the open hole, while no element in the transverse direction reaches the maximum failure value for $0^{\circ}$ layer. Under this load, a small number of elements have failed in the transverse direction and more shear failure are presented around the open hole for the $45^{\circ}$ layer, but no fiber failure occurs in this layer. At $90 \%$ ultimate load, the fiber failure, the progressive degradation of transverse and shear moduli for $0^{\circ}$ layer and $45^{\circ}$ layer all continue to extend. When reaching the ultimate load, all damage contours of $0^{\circ}$ or $45^{\circ}$ layer have spanned the width of the specimen except for the fiber failure of $45^{\circ}$ layer, after which the crack propagates rapidly until most of elements around the open hole are failed. It can be observed the degree of damage for shear modulus are greater than that of transverse modulus in both $0^{\circ}$ and $45^{\circ}$ layers, which is consistent with the theoretical model shown in Figure 4 above. The final damage patterns of $0^{\circ}$ or $45^{\circ}$ layer are in agreement with the experimental results, which proves that the prediction of failure mode is reliable.

Figure 12 shows the nonlinear damage contours of three matrix subcells for $0^{\circ}$ layer and $45^{\circ}$ layer under both $16.7 \mathrm{kN}$ and the ultimate loads using ST/GMC/FF method. Under the loading of $16.7 \mathrm{kN}$, the shear modulus of the second matrix subcell at $0^{\circ}$ layer begins to fail for a few elements at the upper and lower edges of the open hole, while none of the damage of transverse modulus of any element reaches its failure value. The other two matrix subcells for $0^{\circ}$ layer have the same damage evolution and do not reach the lower limit of damage 0.4 . Under this load, the shear elastic moduli $g_{s}$ of the three matrix subcells for $45^{\circ}$ layer have been extended into a cross shape of $\pm 45^{\circ}$, however, just a few transverse failure appears at the upper and lower edges of the open hole for the matrix subcells. The transverse and shear modulus progressive damage patterns are basically the same. Under the ultimate load, the transverse elastic modulus and shear modulus of all subcells in $45^{\circ}$ layer have evolved to the failure value in almost all finite elements around 
the hole, which is not shown Figure 12. The shear moduli of first and third matrix subcells at $0^{\circ}$ layer evolve to the limit value 0.4 for most elements, as shown in the third row of Figure 12, while the damage of transverse elastic moduli evolve along the direction of $x_{2}$ axis, which does not span the finite element model. It can be seen that the elastic modulus of second matrix subcell degrade faster than the other two matrix subcells, because the second matrix subcell locates at the same column with the fiber subcell, which have a large value of stiffness component according to the stress continuity assumption of GMC, i.e., $\sigma_{22}$ in the same column of RUC are equal on the $x_{2}$ axis shown in Figure 2. This phenomenon is very obvious at $0^{\circ}$ layer, but not at $45^{\circ}$ layer, indicating that the reduction evolution of matrix subcells is sensitive to the layer angle.

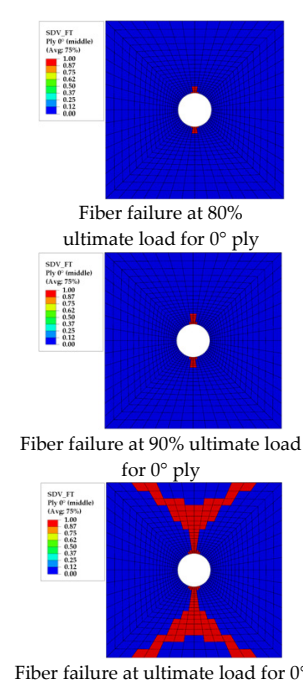

ply
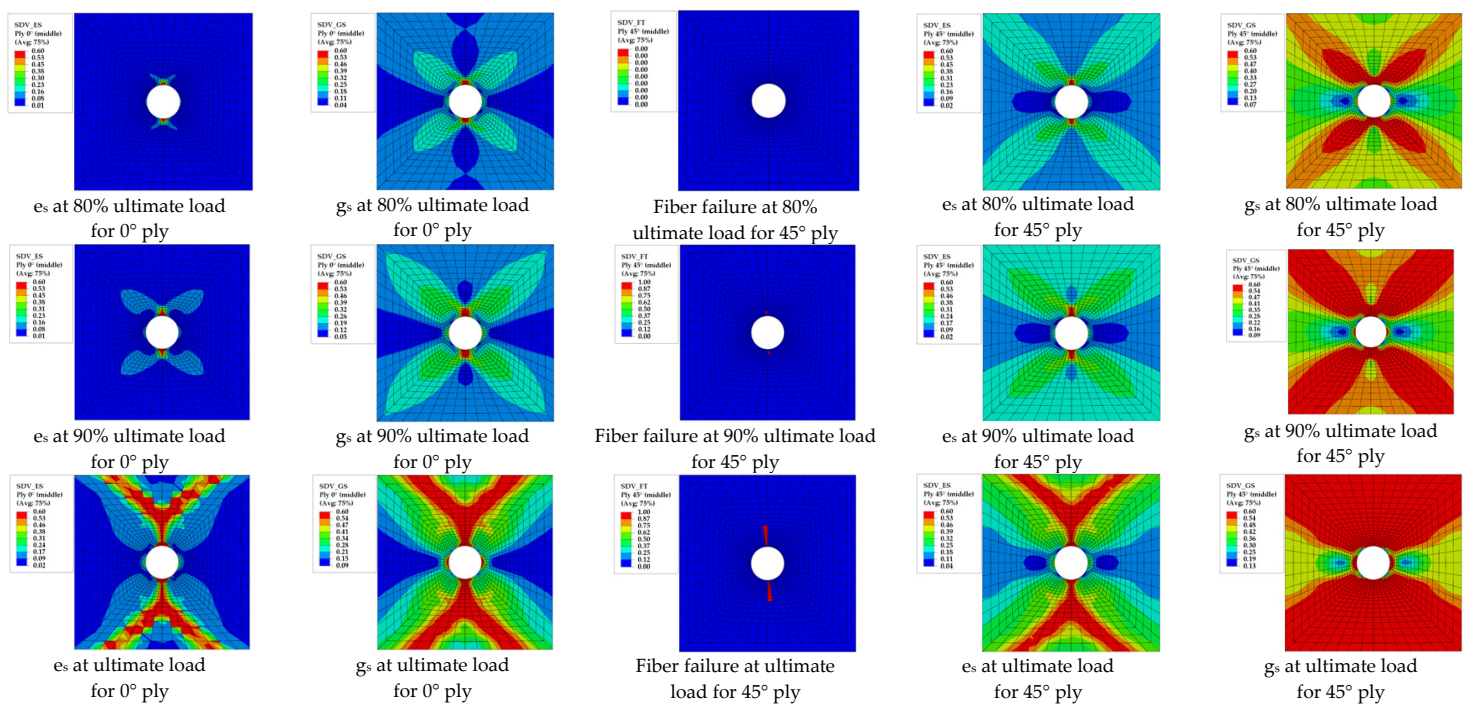

Figure 11. Matrix damage evolution and fiber failure propagation based on ST/FF method.
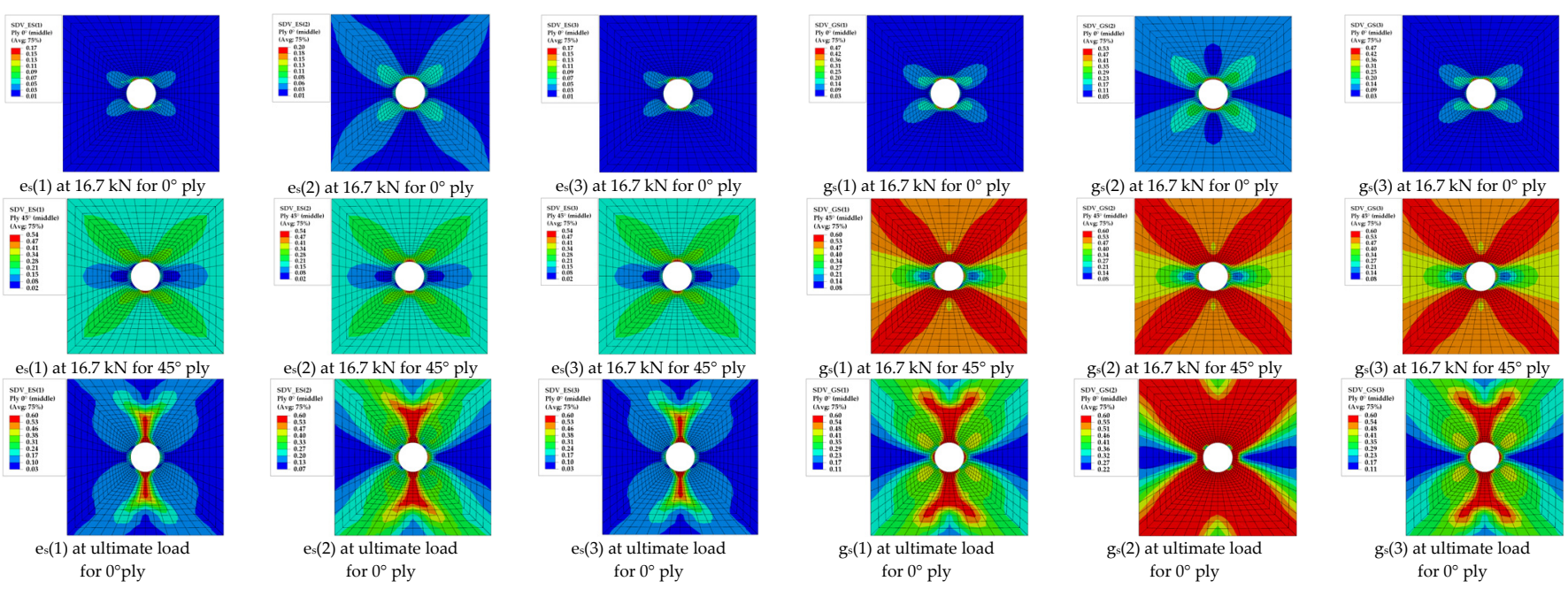

Figure 12. Matrix damage evolution based on ST/GMC/FF method.

As shown in Figure 13, the damage state of $0^{\circ}$ layered fiber subcells is similar to the damage contour obtained by ST/FF method, in which the failure starts from the elements at the upper and lower edges of the hole and expands along the oblique crossing direction, until it runs horizontally through the finite element model. Because the RUC model used contains only one fiber subcell, the failure of the fiber subcell means the fiber failure for the whole RUC at the finite element scale. 


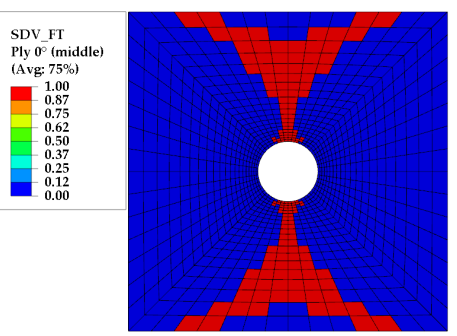

(a)

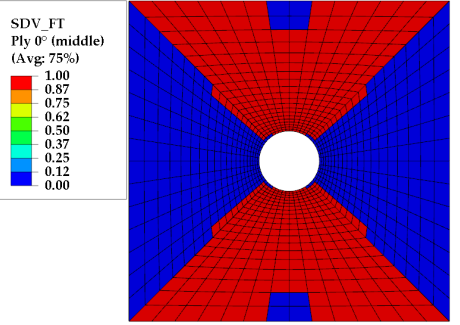

(b)

Figure 13. Failure of fiber subcell. (a) Failure at ultimate load; (b) The final failure.

\section{Conclusions}

By using ST/FF, Hashin progressive damage model and ST/GMC/FF multi-scale progressive damage model, the ultimate load, failure mode and nonlinear damage evolution of fiber reinforced composite laminates with a central open hole were analysed, and the tensile experiment results of 8 specimens were compared. The following conclusions can be drawn:

1. ST/FF nonlinear damage model, Hashin-Rotem progressive damage analysis method, and the ST/GMC/FF model based on the multi-scale progressive damage model are ideal to predict the damage state, failure pattern and the ultimate load, where the ST method well captured the nonlinear behavior of the laminates and reasonable failure mode prediction was obtained. The ST method makes the matrix damage become a natural nonlinear damage evolution and replaces the traditional matrix failure criterion model. Fiber tensile failure shows obvious brittleness behavior, so the failure criterion of fiber is still needed in both macroscopic and microscopic models.

2. Based on the multi-scale progressive damage model of GMC model, ST/GMC/FF methods will resolve stresses or strains of a laminate to the repeating unit cell at the microscopic level, which make the matrix and fiber constituents to be distinguishable, and different matrix subcells also have independent damage evolvement and failure states. It shows that the multi-scale method based on the GMC model can better explain the crack path and failure mechanism for the matrix constituent of a laminate.

Author Contributions: Conceptualization, validation and project administration, M.T.; Methodology, software, investigation and writing — original draft preparation, J.S. All authors have read and agreed to the published version of the manuscript.

Funding: This work was Funded by the Priority Academic Program Development of Jiangsu Higher Education Institutions, National Natural Science Foundation of China (NSFC)(52075232), Natural Science Foundation of Jiangsu Province(BK20201112), and project of "Analysis and Research on Transient Response of Pressure Impulse and Fatigue Life of Hydraulic Product". (201928052009).

Institutional Review Board Statement: Not applicable.

Informed Consent Statement: Not applicable.

Data Availability Statement: The data will be made available on request.

Conflicts of Interest: The authors declare no conflict of interest. The funders had no role in the design of the study; in the collection, analyses, or interpretation of data; in the writing of the manuscript, or in the decision to publish the results.

\section{References}

1. Soden, P.D.; Hinton, M.J.; Kaddour, A.S. Lamina properties, lay-up configurations and loading conditions for a range of fibre-reinforced composite laminates. Compos. Sci. Technol. 1998, 58, 1011-1022. [CrossRef]

2. Soden, P.D.; Hinton, M.J.; Kaddour, A.S. A comparison of the predictive capabilities of current failure theories for composite laminates. Compos. Sci. Technol. 1998, 58, 1225-1254. [CrossRef]

3. Hinton, M.J.; Kaddour, A.S.; Soden, P.D. A comparison of the predictive capabilities of current failure theories for composite laminates, judged against experimental evidence. Compos. Sci. Technol. 2002, 62, 1725-1797. [CrossRef] 
4. Puck, A.; Schürmann, H. Failure analysis of FRP laminates by means of physically based phenomenological models. Compos. Sci. Technol. 2002, 62, 1633-1662. [CrossRef]

5. Pinho, S.; Iannucci, L.; Robinson, P. Physically-based failure models and criteria for laminated fibre reinforced composites with emphasis on fibre kinking: Part I: Development. Compos. Part A Appl. Sci. Manuf. 2006, 37, 63-73. [CrossRef]

6. Icardi, U.; Locatto, S.; Longo, A. Assessment of recent theories for predicting failure of composite laminates. Appl. Mech. Rev. 2007, 60, 76-86. [CrossRef]

7. Beaumont, P.; Dimant, R.A.; Shercliff, H.R. Failure processes in composite materials: Getting physical. J. Mater. Sci. 2006, 41, 6526-6546. [CrossRef]

8. Mehrmashhadi, J.; Chen, Z.; Zhao, J.; Bobaru, F. A stochastically homogenized peridynamic model for intraply fracture in fiber-reinforced composites. Compos. Sci. Technol. 2019, 182, 107770. [CrossRef]

9. Mehrmashhadi, J.; Tang, Y.; Zhao, X.; Xu, Z.; Pan, J.; Le, Q.V.; Bobaru, F. The effect of solder joint microstructure on the drop test failure-a peridynamic analysis. IEEE Trans. Compon. Packag. Manuf. Technol. 2018, 9, 58-71. [CrossRef]

10. Tallia, R.; Morello, P.; Castellano, G. Damage and failure of composite materials. AIAA J. 2012, 52, 317-323.

11. Hallett, S.R.; Green, B.G.; Jiang, W.G.; Wisnom, M.R. An experimental and numerical investigation into the damage mechanisms in notched composites. Compos. Part A Appl. Sci. Manuf. 2009, 40, 613-624. [CrossRef]

12. Camanho, P.P.; Erçin, G.H.; Catalanotti, G.; Mahdi, S.; Linde, P. A finite fracture mechanics model for the prediction of the open-hole strength of composite laminates. Compos. A Appl. Sci. Manuf. 2012, 43, 1219-1225. [CrossRef]

13. Pierron, F.; Green, B.; Wisnom, M.R.; Hallet, S.R. Full-field assessment of the damage process of laminated composite open-hole tensile specimens. Part II Exp. Results Compos. Part A Appl. Sci. Manuf. 2007, 38, 2321-2332. [CrossRef]

14. Orifici, A.C.; Herszberg, I.; Thomson, R.S. Review of methodologies for composite material modelling incorporating failure Compos. Struct. 2008, 86, 194-210. [CrossRef]

15. Sadeghi, G.; Hosseini-Toudeshky, H.; Mohammadi, B. An investigation of matrix cracking damage evolution in composite laminates-development of an advanced numerical tool. Compos. Struct. 2014, 108, 937-950. [CrossRef]

16. Iarve, E.V.; Mollenhauer, D.; Kim, R. Theoretical and experimental investigation of stress redistribution in open hole composite laminates due to damage accumulation. Compos. Part A Appl. Sci. Manuf. 2005, 36, 163-1717. [CrossRef]

17. Talreja, R. Multi-scale modeling in damage mechanics of composite materials. J. Mater. Sci. 2006, 41, 6800-6812. [CrossRef]

18. Paley, M.; Aboudi, J. Micromechanical analysis of composites by the generalized cells model. Mech. Mater. 1992, 14, 127-139. [CrossRef]

19. Pindera, M.J.; Bednarcyk, B.A. An efficient implementation of the generalized method of cells for unidirectional, multi-phased composites with complex microstructures. Compos. Part B-Eng. 1999, 30, 87-105. [CrossRef]

20. Naghipour, P.; Arnold, S.M.; Pineda, E.J.; Stier, B.; Hansen, L.; Bednarcyk, B.A.; Waas, A.M. Multiscale static analysis of notched and unnotched laminates using the generalized method of cells. J. Compos. Mater. 2016, 51, 1433-1454. [CrossRef]

21. Shi, J.; Tong, M.; Zhou, C.; Ye, C.; Wang, X. Progressive Failure Analysis in Open-Hole Tensile Composite Laminates of Airplane Stringers Based on Tests and Simulations. Appl. Sci. 2020, 11, 185. [CrossRef]

22. Schapery, R.A. A theory of mechanical behavior of elastic media with growing damage and other changes in structure. J. Mech Phys. Solids 1990, 38, 215-253. [CrossRef]

23. Schapery, R.A. Prediction of compressive strength and kink bands in composites using a work potential. Int. J. Solids. Struct. 1995, 32, 739-765. [CrossRef]

24. Lamborn, M.J.; Schapery, R.A. An investigation of the existence of a work potential for fiber-reinforced plastic. J. Compos. Mater. 1993, 27, 352-382. [CrossRef]

25. Sicking, D.L. Mechanical Characterization of Nonlinear Laminated Composites with Traverse Crack Growth; Texas AM University College: College Station, TX, USA, 1992.

26. Pineda, E.J.; Waas, A.M. Numerical implementation of a multiple-ISV thermodynamically-based work potential theory for modeling progressive damage and failure in fiber-reinforced laminates. Int. J. Fract. 2013, 182, 93-122. [CrossRef] 